Frazier, R. A., W. J. Duncan, and A. R. Collier, Elementary Matrices, Cambridge Univ. Press, England (1955).

Newbold, F. R., and N. R. Amundson, "A Model for Evaporation of a Multicomponent Droplet," AIChE J., 19, 22 (1973).

Swamy, M. N. S., "On a Formula for Evaluating $e^{A} t$ when the
Eigenvalues of A are not Necessarily Distinct," Matrix Tensor Quarterly, 23, 67 (1972).

Manuscript received May 14, 1973; revision recelved June 21 and accepted July 23, 1973.

\title{
Note on Light Transmission through a Polydisperse Dispersion
}

RANE L. CURL

Department of Chemical Engineering

University of Michigan

Ann Arbor, Michigan 48104

A recent paper of McLaughlin and Rushton (1973) presented an analysis of light transmission through a dense dispersion of spherical particles when only the unscattered light is received by the detector. They numerically generated samples from various drop size distributions and, using a relation for the probability of a light ray (in a parallel beam) not striking any of the drops, found that the total light transmission is a unique exponential function of the group $a l$, where $a$ is the interfacial area per unit volume, and $l$ is the path length through the dispersion. They confirmed this numerically for four different drop size distributions. It may be shown that this result is theoretically exact for all drop size distributions, subject to certain idealizations. Actually, Calderbank (1958) showed this, in a rather elegant fashion, but used a distribution-free argument that obscured the fact that the result is independent of a drop size distribution. The Calderbank equation is equal to that obtained by McLaughlin and Rushton. The following derivation simplifies but generalizes the analyses of Otvos et al. (1957) and Gumprecht and Sliepcevich (1953a, 1953b).

Assuming, as did McLaughlin and Rushton, a beam of parallel rays, and that scattered light (diffracted or refracted) is not received by the detector, the effective scattering cross section of a single drop of radius $r$ is

$$
K_{a} \pi r^{2}
$$

where $K_{a}$ is the total-scattering coefficient. If the path length is $l$, the number of particles per unit volume is $n$, and their probability density distribution of radius $r$ is $p(r)$, the average number of drops $\bar{q}$ that lie along any particular path is

$$
\bar{q}=\int_{0}^{\infty} K_{a} \pi r^{2} n l p(r) d r
$$

Since $4 \pi r^{2} N p(r) d r$ is just the interfacial area per unit volume contributed by drops having radii between $r$ and $r+d r$, if $K_{a}$ is a constant (an acceptable assumption for large drops when also $K_{a}=1$ ), this becomes

$$
\bar{q}=\frac{1}{4} K_{a} a l
$$

We now assume that the drops occur randomly and independently on a light path. The number present is then Poisson distributed (this assumes no interference between drops, as did McLaughlin and Rushton), or

$$
p(q)=\frac{\bar{q}^{q} \exp (-\bar{q})}{q !}
$$

The probability of light transmission on a path, and therefore in a beam, is the probability that $q=0$, or $\exp (-\bar{q})$. Combining this and Equation (3), and letting $K_{a}=1$, we obtain for the light attenuation

$$
f=\exp \left(-\frac{1}{4} a l\right)
$$

Defining $N_{T}$ as al/6, as did McLaughlin and Rushton, the group in the exponential in Equation (5) is just $1.5 N_{T}$, which was found numerically by them.

\section{NOTATION}

$a \quad=$ interfacial area per unit volume of dispersion

$f \quad=$ ratio of received to incident light intensity, or the probability of light not being scattered from a path

$K_{a}=$ total scattering coefficient

$l \quad=$ length of light path through dispersion

$n \quad=$ number of drops per unit volume of dispersion

$N_{T} \quad=$ transmission number of McLaughlin and Rushton

$p(r)=$ probability density distribution of drop radius

$p(q)=$ probability of there being $q$ drops on a light path

$q \quad=$ number of drops on a light path

$\bar{q} \quad=$ average number of drops on a light path

$r \quad=$ drop radius

\section{LITERATURE CITED}

Calderbank, P. H., "Physical Rate Processes in Industrial Fermentation, Part I: The Interfacial Area in Gas-Liquid Contracting with Mechanical Agitation," Trans. Instn. Chem. Engrs., 36, 443 ( 1958).

Gumprecht, R. O., and C. M. Sliepcevich, "Scattering of light by large spherical particles," J. Phys. Chem., 57, 90 (1953).

-." "Measurements of particle sizes in polydispersed systems by means of light transmission combined with differential settling," ibid., 57, 95 (1953).

McLaughlin, C. M., and J. H. Rushton, "Interfacial Areas of Liquid-Liquid Dispersions from Light Transmission Measurements," AIChE J., 19, 817 (1973).

Otvos, J. W., H. Stone, and W. R. Harp, "Theory of Radiant Energy Absorption by Randomly Dispersed Discrete Particles," Spectrochimica Acta, 9, 148 (1957).

Manuscript received August 10, 1973, and accepted September 4, 1973. 